\title{
Macrophage polarization in interstitial lung diseases
}

\author{
PAWE€ WOJTAN ${ }^{1}$, MICHAE MIERZEJEWSKI ${ }^{1}$, IWONA OSINSSKA ${ }^{2}$, \\ JOANNA DOMAGAEA-KULAWIK ${ }^{1}$
}

${ }^{1}$ Department of Internal Medicine, Pneumonology and Allergology, Medical University of Warsaw, Poland

${ }^{2}$ Department of Pathology, Medical University of Warsaw, Poland

\begin{abstract}
The role of bronchoalveolar lavage fluid (BALf) examination in differential diagnosis of interstitial lung diseases (ILD) was established. Currently, functional polarization into M1 (pro-inflammatory) and M2 (anti-inflammatory) subpopulations is emphasized. ILD.

The aim of our study was to compare the proportion of $M 1$ and $M 2$ in BALf of patients with different

BALf samples were collected from 75 ILD patients: sarcoidosis (SA, 36), hypersensitivity pneumonitis (HP, 10), non-specific interstitial pneumonia (NSIP, 8), idiopathic pulmonary fibrosis (IPF, 6) and other ILD (15). Phenotyping was performed by immunocytochemistry with anti-CD40 and CD163 antibodies (for $M 1$ and $M 2$, respectively).

For both, CD40 and CD163, three populations of cells have been specified: small cells with strong $(+++)$, large cells with weak $(+)$ and cells with no (-) reaction. Due to lack of statistically significant differences between patients with HP, NSIP and IPF, they were classified into a common group and compared to the group of patients with sarcoidosis. The median proportion of macrophage population was as follows: for CD40: $61 \%, 35 \%, 2 \%$ in patients with $S A$ and $49 \%, 47 \%$, 3\% in patients with other ILD and for CD163: 55\%, 35\%, 5\% in SA and 53\%, 43\%, $1 \%$ in ILD patients, respectively. We found a significantly higher proportion of M1 in SA when compared with other ILD.

Our study showed no evidence of defined polarization of alveolar macrophages in different types of interstitial lung diseases. However, we emphasized the role of CD40 positive cells in sarcoidosis and the role of CD163 positive cells in fibrotic diffuse lung diseases.
\end{abstract}

Key words: sarcoidosis, interstitial lung disease, BAL, macrophages $M 1$, macrophages M2, CD4O, CD163.

(Cent Eur J Immunol 2016; 41 (2): 159-164)

\section{Introduction}

Bronchoalveolar lavage (BAL) is the diagnostic and therapeutic procedure performed during bronchoscopy, which plays an established role in differential diagnosis of lung diseases, including interstitial lung diseases (ILD) [1, 2]. BAL fluid (BALf) allows to obtain cells and extracellular material and cells retrieved by BAL are macrophages, lymphocytes, neutrophils and eosinophils [2]. The relative and absolute amounts of these cells differ in various diseases [3]. Among cells retrieved by BAL, macrophages are the most numerous population, but their role in pathogenesis of ILD, apart from sr-ILD, was not well established [4]. For many decades macrophages have been perceived as a uniform cell population, but currently functional po- larization into M1 (pro-inflammatory or classically activated) and M2 (anti-inflammatory or alternatively activated) subpopulations is emphasized. First studies describing classical activation of macrophages come from 1960s [5]. Polarization towards M1 macrophages depends on bacterial lipopolysaccharide (LPS) and agents produced by activated T helper 1 lymphocytes (Th1 lymphocytes) and natural killer (NK) cells, particularly interferon $\gamma($ IFN- $\gamma$ ) and other proinflammatory cytokines such as tumor necrosis factor $\alpha$ (TNF- $\alpha$ ) [6-10]. M1 macrophages produce proinflammatory cytokines such as TNF- $\alpha$ and interleukin 12 (IL-12) [7]. They have cytotoxic properties and the ability to kill intracellular parasites [6, 7]. In turn, M2 macrophages are induced by Th2 lymphocytes-related cytokines: IL-4, IL-13 and immune complexes and glucocorticoste-

Correspondence: Paweł Wojtan, Department of Internal Medicine, Pneumonology and Allergology, Medical University of Warsaw, Poland, e-mail: wojtan.pawel@gmail.com

Submitted: 27.07.2015, Accepted: 13.11.2015 
roid hormones [7]. They release inflammatory mediators such as IL-10. They have immunoregulatory functions, are involved in angiogenesis and wound healing. These two populations have different surface markers, allowing to distinguish them from each other with specific antibodies against the markers. CD163 molecule has been shown as a marker of macrophages with anti-inflammatory properties - M2 cells [8, 11-14]. M1 detection is more controversial because there is no single, widely accepted marker. Used methods include surface markers such as CD40 and CD86 [8, 9, 14-17], expression of nitric oxide synthase 2 (NOS-2, also denoted iNOS) $[6,18]$ or human leukocyte antigen (HLA) [8, 10, 15]. Monocyte polarization towards M1 or M2 is not a definitive process. Plastic gene expression allows macrophages to switch from one population to another as a response to changes in the microenvironment. Chemokines attract macrophages that interact with other host cells (particularly T cells), neoplastic cells, microorganisms and that microenvironmental stimulation could cause a shift from M1 to M2 subpopulation [19]. The aim of our study was to describe the alveolar macrophage phenotype in ILD by analysis of BALf cells using immunocytochemistry with anti-CD40 and CD163 antibodies.

\section{Material and methods}

We analyzed retrospectively BAL samples of patients who underwent the diagnostic procedure. The study was conducted in 2013/2014. 75 subjects ( 36 female, 39 male) with the following interstitial lung diseases: sarcoidosis, stages I-III (SA, $n=36$ ), hypersensitivity pneumonitis (HP, $n=10$ ), non-specific interstitial pneumonia (NSIP, $n=8$ ), idiopathic pulmonary fibrosis (IPF, $n=6$ ) and other ILD $(n=15)$ were enrolled in the study (classified according to [20]). In the last group the following diseases were included: desquamative interstitial pneumonia (DIP), cryptogenic organizing pneumonia (COP), chronic eosinophilic pneumonia (CEP) and systemic sclerosis-associated interstitial lung disease (SSc-ILD). The mean age of patients was 49.7 years (range 26-83) and was comparable in each group. There were $37 \%$ of smokers and $52 \%$ of never smokers in the study group, in $11 \%$ of cases no data were available. During enrolment of the study group we excluded patients with neoplastic diseases, asthma and chronic obstructive pulmonary disease (COPD) and treated with corticosteroids and/or immunosuppressive drugs. All diagnoses were established in accordance with proper guidelines. The study was approved by the Bioethical Committee at Medical University of Warsaw. Bronchoscopy and BAL were performed during the diagnostic process. Sampling, processing and analysis of the collected material was based on the guidelines developed by the experts of the Polish Respiratory Society [2] that are consistent with recommendations of the American Thoracic Society [1].

\section{Immunocytochemistry}

Four slides from each subject were analyzed. Two of them were stained with May-Grunwald-Giemsa method and used to determine the differential cell count. Phenotyping was performed on the next two slides by immunocytochemistry with anti-CD40 and CD163 antibodies (AbD Serotec, Raleigh, North Carolina, USA), for M1 and M2, respectively. Material has been evaluated under the microscope at a $1000 \times$ magnification and $300 \mathrm{immu}-$ nolabeled macrophages on each slide were then counted by two independent pathologists experienced in pneumonological cytology. Initially, we confirmed the specificity of this staining on macrophage culture obtained from cells isolated from BALf and stimulated towards M1 and M2 using selected mediators. The staining technique was precisely described in our previous paper [17].

\section{Statistical analysis}

Statistical analysis was performed with Statistica software (StatSoft, Poland, version 10.0). Mann-Whitney $U$ test was used to compare two groups, for more than two group comparison, the Kruskal-Wallis test (for data non-normally distributed) was applied. Differences were considered significant when $p<0.05$.

\section{Results}

The BAL cellular pattern is presented in Table 1 - the changes are typical - patients with SA and particularly HP and NSIP present a lymphocytic cellular pattern and patients with IPF present a neutrophilic pattern. For both, CD40 and CD163 antigens staining, three populations of cells have been specified: small cells with strong $(+++)$, large cells with weak $(+)$ and cells with no (-) reaction (Fig. 1). We confirmed this by statistical analysis - it was a significant reverse correlation between the proportion of high $(+++)$ and low $(+)$ intensity of reaction (Spearman test, $r>0.4, p<0.05)$. The median proportion and interquartile range of these macrophage populations in each ILD group are presented in Table 2. We did not find any significant difference when compared these groups. Due to the small size of groups of patients with various stages of sarcoidosis, statistical analysis within this group was not possible. Due to lack of statistically significant differences between patients with HP, NSIP and IPF, they were classified into a common group and compared to the group of patients with sarcoidosis (Fig. 2). We found a significantly higher proportion of M1 cells in SA when compared with other ILD (CD40+++ $61 \%$ vs. $49 \%, p<0.05)$, however CD $40+$ cells were more numerous in the ILD group $(35 \%$ vs. $47 \%, p<0.05)$. The proportion of CD163- was significantly higher in the SA group $(5 \%$ vs. $1 \%, p<0.05)$. The proportion of CD163+ cells was elevated in the group of ILD, but without significance ( $43.6 \%$ vs. $34.6 \%$ ). 
Table 1. BALf cellular pattern in all investigated groups of patients with interstitial lung diseases

\begin{tabular}{lcccccc}
\hline & Overall & Sarcoidosis & $\begin{array}{c}\text { Interstitial } \\
\text { lung diseases } \\
\text { combined }\end{array}$ & $\begin{array}{c}\text { Hypersensitivity } \\
\text { pneumonitis }\end{array}$ & $\begin{array}{c}\text { Nonspecific } \\
\text { interstitial } \\
\text { pneumonia }\end{array}$ & $\begin{array}{c}\text { Idiopathic } \\
\text { pulmonary } \\
\text { fibrosis }\end{array}$ \\
\hline Total cell count, $\times 10^{6}$ & $18(12-31)$ & $17(12-24)$ & $19(12-32)$ & $25(18-32)$ & $20(17-26)$ & $12(9-16)$ \\
\hline Alveolar macrophages, $\times 10^{6}$ & $8(4-15)$ & $9(6-15)$ & $6(3-14)$ & $5(3-12)$ & $4(3-12)$ & $8(6-11)$ \\
\hline Alveolar macrophages, $\%$ & $51(31-74)$ & $51(41-76)$ & $47(20-68)$ & $18(17-39)$ & $25(22-27)$ & $70(64-76)$ \\
\hline Lymphocytes, $\times 10^{6}$ & $5(2-11)$ & $5(2-8)$ & $5(2-12)$ & $12(9-18)$ & $14(8-15)$ & $1(1-2)$ \\
\hline Lymphocytes, $\%$ & $35(13-47)$ & $37(18-46)$ & $31(12-63)$ & $63(41-78)$ & $67(42-75)$ & $11(9-14)$ \\
\hline Neutrophils, $\times 10^{6}$ & $1(1-2)$ & $1(0-1)$ & $1(1-3)$ & $2(1-5)$ & $1(1-5)$ & $2(1-2)$ \\
\hline Neutrophils, \% & $6(4-11)$ & $4(3-8)$ & $7(4-17)$ & $7(6-15)$ & $5(3-18)$ & $16(14-18)$ \\
\hline Eosinophils, $\times 10^{6}$ & $0(0-1)$ & $0(0-1)$ & $0(0-1)$ & $0(0-1)$ & $0(0-0)$ & $0(0-1)$ \\
\hline Eosinophils, $\%$ & $2(1-3)$ & $2(1-3)$ & $2(0-5)$ & $1(1-3)$ & $1(0-3)$ & $3(3-3)$ \\
\hline Data are presented as median $\left(\right.$ IQR $25-75^{\text {th }}$ percentile). & & & & & &
\end{tabular}
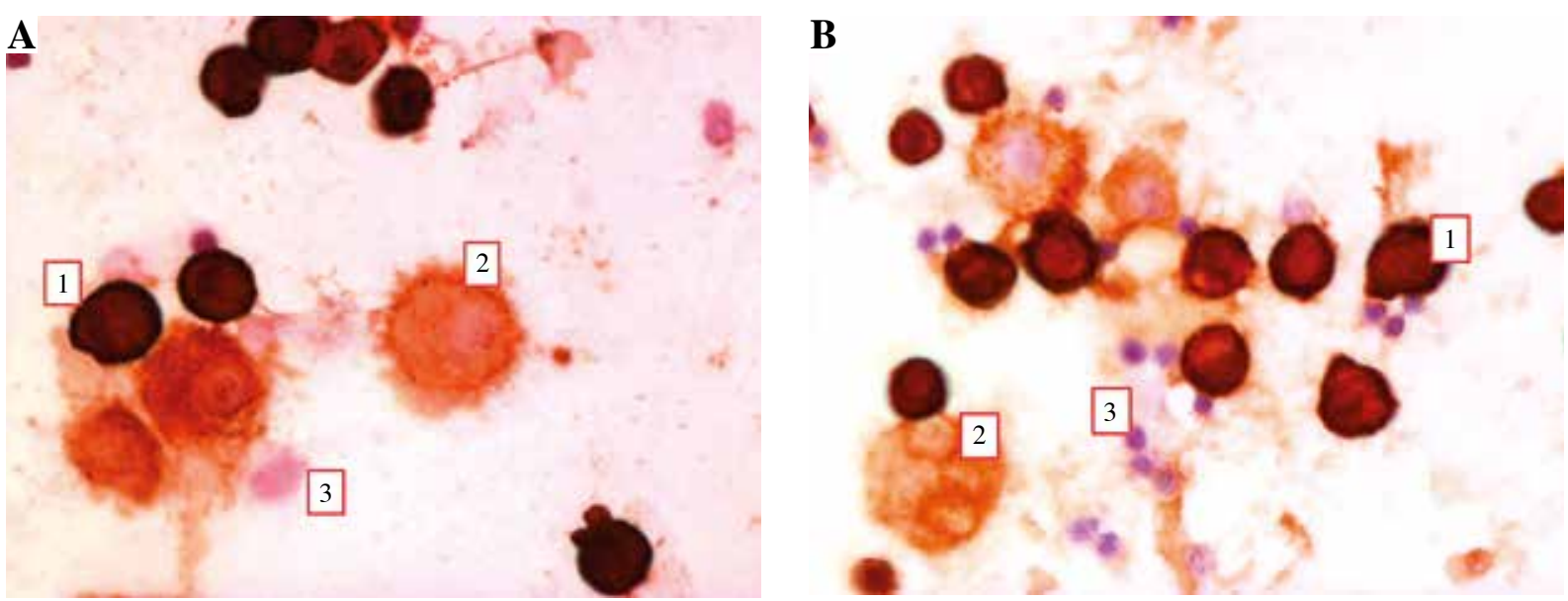

Fig. 1. Macrophages stained with CD40 (A) and CD163 (B). 1 - small cells with strong reaction, 2 - big cells with weak reaction, 3 - cells with no reaction

We found some significant correlation of macrophages with CD40 and CD163 reaction. There was a significant correlation of CD40+++ with CD163+++ cells $(r=0.25$, $p<0.05)$ and a strong negative relation of CD40+++ cells with those with weak reaction CD40+ $(r=-0.9$, $p<0.05)$. Similarly in the population of CD163 positive cells (CD163+++ with CD163+, $r=-0.8, p<0.05$ ). There was a positive correlation between the proportion of CD40-positive cells with the number of lymphocytes in the sarcoidosis group.

In spite of a low number of patients we separately analyzed three cases of DIP. The results of this analysis are presented in Table 3. A very low number of small cells with strong reaction with CD40 and CD163 was noticed.

\section{Discussion}

In this study we evaluated two types of macrophages in a relatively large group of ILD patients. We used the immunocytochemistry method to assess macrophages with an expression of CD40 (the marker of M1 differentiation) and CD163 as a marker for M2 cells. We observed two types of cells: one type with strong and a second with weak reaction to these two markers without any significant difference between different types of ILD apart from a significantly elevated proportion of CD40+++ cells in sarcoidosis.

Our choice of immunocytochemistry for cell phenotype evaluation was dictated by the accuracy of this technique in identification of alveolar macrophage and the differentiation them from other immune cells. Moreover, it was possible to perform the analysis in the archived previously stored samples. The cells with strong, weak or no reaction were well recognized and there was high repeatability between observers in our study. Macrophages are the cells with many different, often contradictory functions. The functional phenotypes of monocyte/macrophages were recently recognized: activated, wound-heal- 
Table 2. Proportion of macrophages with an expression of CD40 and CD163 in the BAL fluid of patients with interstitial lung diseases (ILD)

\begin{tabular}{lcccccc}
\hline & Overall & Sarcoidosis & $\begin{array}{c}\text { Interstitial } \\
\text { lung diseases } \\
\text { combined }\end{array}$ & $\begin{array}{c}\text { Hypersensitivity } \\
\text { pneumonitis }\end{array}$ & $\begin{array}{c}\text { Nonspecific } \\
\text { interstitial } \\
\text { pneumonia }\end{array}$ & $\begin{array}{c}\text { Idiopathic } \\
\text { pulmonary } \\
\text { fibrosis }\end{array}$ \\
\hline $\mathrm{CD} 40(+++) \%$ & $56(44-67)$ & $61(55-69) *$ & $49(35-57)^{*}$ & $54(51-63)$ & $46(37-63)$ & $52(46-66)$ \\
\hline $\mathrm{CD} 40(+), \%$ & $41(31-51)$ & $35(28-40)^{*}$ & $47(42-54)^{*}$ & $44(39-47)$ & $51(37-59)$ & $45(33-49)$ \\
\hline $\mathrm{CD} 40(-), \%$ & $2(1-4)$ & $2(1-4)$ & $3(1-5)$ & $2.5(2-4)$ & $3(1-4)$ & $2(1-4)$ \\
\hline $\mathrm{CD} 163(+++), \%$ & $55(46-63)$ & $55.5(49-63)$ & $53(44-63)$ & $56(51-76)$ & $53(48-59)$ & $58(39-76)$ \\
\hline $\mathrm{CD} 163(+), \%$ & $40(27-49)$ & $35(27-47)$ & $43(27-54)$ & $40(18-43)$ & $43(39-50)$ & $36(22-60)$ \\
\hline $\mathrm{CD} 163(-), \%$ & $3(1-7)$ & $5(3-10) *$ & $1(1-3) *$ & $1(1-3)$ & $3(2-4)$ & $1.5(0-7)$ \\
\hline Data are presented as median $\left(\right.$ IQR $25-75^{\text {th }}$ percentile $) *$ difference significant in Kruskal-Wallis test $(p<0.05)$. & &
\end{tabular}

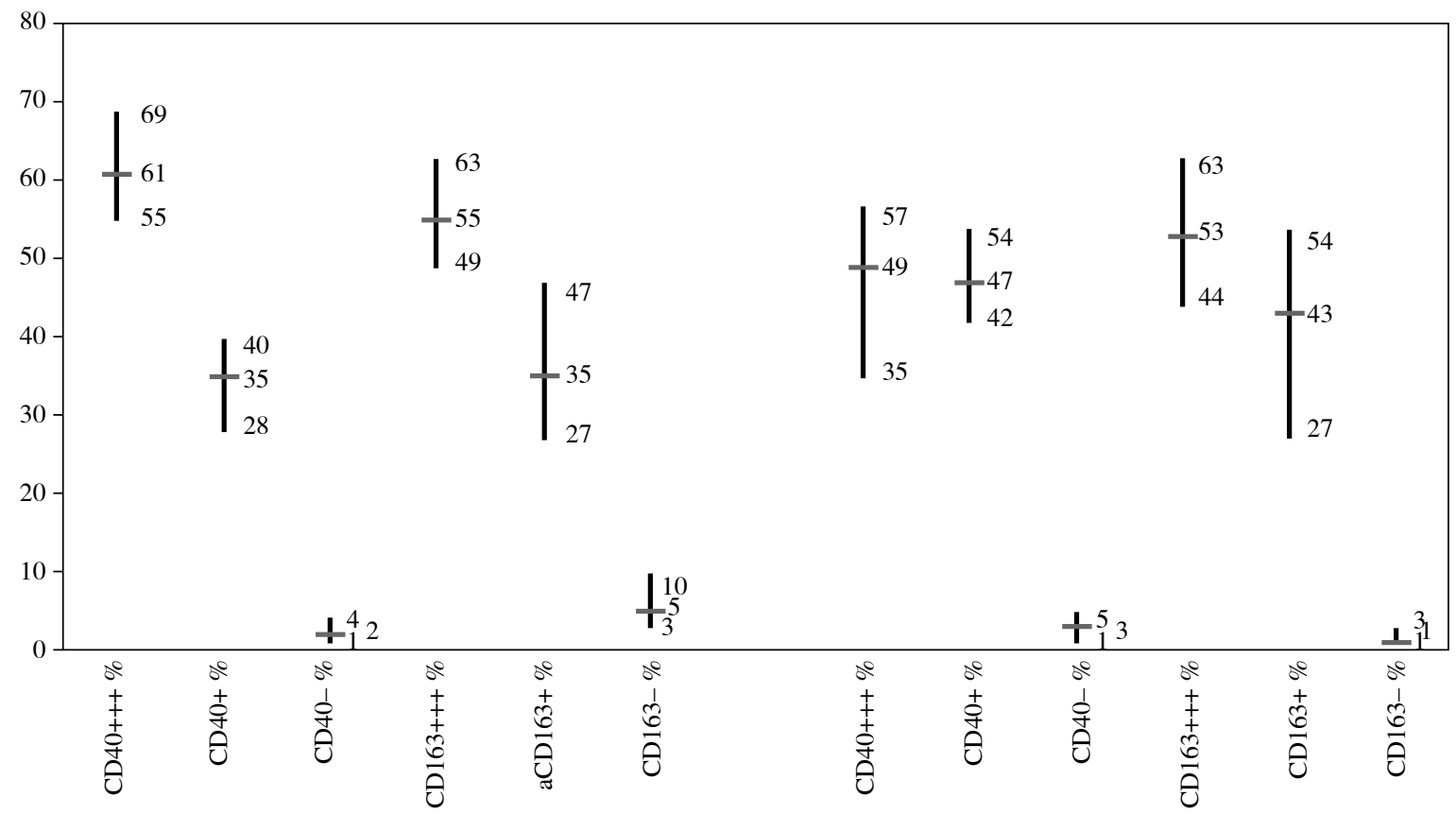

Fig. 2. The median proportion of BALf macrophages with expression of CD40 and CD163 in sarcoidosis and ILD groups

ing and regulatory [21]. The division into two populations (M1 and M2) is a kind of simplification, because in vivo the individual cells have different expression of their surface molecules [22]. The summation of the percentages of cells stained with anti-CD40 and anti-CD163 to above $100 \%$ indicates the presence on the cell, of both markers M1 and M2. There was a very low proportion of unstained cells. This means that the cell does not belong strictly to subpopulation but rather is at some intermediate stage of polarization between pro- and anti-inflammatory populations. According to recent data the switch from M1 to M2 is more probable than the reverse/opposite [21]. Evaluation of double-labeled cells by other techniques, for example in the confocal microscopy would confirm this. "Perfect" extremely polarized macrophages may be obtained by stimulating with appropriate agents (e.g. IFN- $\gamma$ for M1 and M-CSF for M2). The limitation of our study was the use of only one method for alveolar macrophages phenotyping. However, our choice of CD40 and CD163 was supported by a well-known function in immune reactions [12, 23].

Our observation of two morphologically distinct populations of macrophages needs a short explanation. The small cells with very strong reaction and large cells with lower one were noticed in population of CD40 positive and CD163 positive macrophages as well. More other/ Moreover there was an adverse significant relation between these two forms suggesting that one could displace another. Taking into account the possible origin of alveo- 
Table 3. Number and proportion of macrophages and proportion of CD40 positive and CD163 positive cells in the BALf of patients with desquamative interstitial pneumonia (DIP)

\begin{tabular}{lccccccc}
\hline $\begin{array}{c}\text { Total cell count } \\
\times 10^{6}\end{array}$ & $\begin{array}{c}\text { Macrophages } \\
\times 10^{6}\end{array}$ & $\begin{array}{c}\text { Macrophages } \\
\%\end{array}$ & $\begin{array}{c}\text { CD } 40(+++), \\
\%\end{array}$ & $\begin{array}{c}\text { CD } 40(+), \\
\%\end{array}$ & $\begin{array}{c}\text { CD163 (+++), } \\
\%\end{array}$ & $\begin{array}{c}\text { CD163 (+), } \\
\%\end{array}$ \\
\hline Case 1 & 30.8 & 26.18 & 85 & 35 & 60 & 39 & 59 \\
\hline Case 2 & 44 & 39.60 & 90 & 29 & 71 & 25 & 73 \\
\hline Case 3 & 33.12 & 19.9 & 60 & 56 & 41 & 38 & 62 \\
\hline Data presented as median. & & & & & & &
\end{tabular}

lar macrophages from monocytes line we assume that the small cells with strong reaction are young and active cell line [21]. The message for further studies could be as follows: the small cells are functionally important and play a role of a guide in the inflammatory process. The large cells seem to be adult, just old preapoptotic form of macrophages [24]. Interestingly, we found a very low proportion of small cells with strong reaction in the BALf of cases with DIP. The role of macrophages in the pathogenesis of DIP was documented and the augmentation of pigmented cells in the BALf is pathognomonic for this entity [4]. The novelty of our recent study is to show a significantly low proportion of small cells with strong CD40 and CD163 reaction and an elevated proportion of large cells with weak reaction in the BALf with DIP pattern. Further studies are needed for explanation of this observation.

There is a small number of published studies dedicated to analysis of BAL macrophages phenotype [16, 25-28]. In the study of Kunz et al., the proportion of CD163+ cells in the BALf was comparable with our results [16]. The authors observed the influence of smoking on polarization to $\mathrm{M} 2$ in the BALf of patients with chronic obstructive lung disease (COPD). In the study of Hodge et al., the functional analysis of BAL macrophages of smokers with COPD was performed without any evidence for definitive polarization of macrophages [25]. We did not confirm any changes of macrophage phenotype in smokers in this study and in our previous work [17]. Admittedly in patients with sarcoidosis the proportion of CD163 negative cells was lower in smokers when compared with non-smokers. In the present study this trend has been continued with no significantly higher proportion of CD163 positive cells in smokers.

In the study of Redente et al., macrophages were harvested from BALf in an experimental model of tuberculosis and the predominance of M1 was found [27]. Finally we found one study concerning the phenotype of macrophages in sarcoidosis presented by Wikén et al. [28]. In this study, the functional tests for macrophage characteristics were used but without any evidence for predominance of any subpopulation [28]. Similar results were reported by Misson et al. [29].
We found an elevated proportion of small cells with strong reaction with CD40 marker in the BALf of patients with sarcoidosis what was different when compared with other ILD. CD40 receptor plays a role in signal transduction during antigen presentation and initiation of immune reaction among others in autoimmune diseases [23]. The contribution of CD40-CD152 pathway to adaptive immune response justifies the usefulness of this marker for M1 detection.

In fibrotic lung disorders, the M2 polarization is suspected. The Th2 pathway with an elevated CCL-18 (marker of M2) concentration in the BALf is linked to the extent of fibrosis in ILD [30]. As it was emphasized in recent studies, the direction of activation and differentiation of $\mathrm{T}$ cell is initiated by macrophages, thus the appropriate macrophage population is capable of promoting Th1 or Th2 response [31]. In the studies on ILD pathogenesis, macrophages seem to remain in the shadows. In the study of Pechkovsky et al. [26], M2 macrophages were identified by cytokine release and the phenotyping by flow cytometry with antibody anti-CD206. Authors concluded that there is evidence for M2 polarization in fibrotic lung diseases. We used CD163 for M2 description. CD163 is a transmembrane protein expressed on monocyte/macrophage cell lines. This expression increases during resolution of inflammation and the process of wound healing [12]. These processes underlie the pathological immune reaction in the course of ILD. Thus, our observation pointed to the role of macrophages with an expression of CD163 and the CD163 molecule in pathogenesis of ILD [32].

In conclusion, the results of our study underline usefulness of immunocytochemistry in evaluation of the macrophage phenotype in the BALf. No evidence of defined polarization of alveolar macrophages in different types of interstitial lung diseases was found. However, we emphasized the role of CD40 positive cells in sarcoidosis and the role of CD163 positive cells in fibrotic diffuse lung diseases.

The authors declare no conflict of interest.

This work was supported by Medical University of Warsaw, grant number: 1WU/NM1/13. 


\section{References}

1. Meyer KC, Raghu G, Baughman RP, et al. (2012): An official American Thoracic Society clinical practice guideline: the clinical utility of bronchoalveolar lavage cellular analysis in interstitial lung disease. Am J Respir Crit Care Med 185: 1004-1014.

2. Chcialowski A, Chorostowska-Wynimko J, Fal A, et al. (2011): Recommendation of the Polish Respiratory Society for bronchoalveolar lavage (BAL) sampling, processing and analysis methods. Pneumonol Alergol Pol 79: 75-89.

3. Domagala-Kulawik J, Skirecki T, Maskey-Warzechowska M, et al. (2012): Bronchoalveolar lavage total cell count in interstitial lung diseases-does it matter? Inflammation 35: 803809.

4. Domagala-Kulawik J, Maskey-Warzechowska M, Krenke R, et al. (2008): Role of bronchoalveolar lavage in the initial diagnosis of smoking-related interstitial lung diseases. J Physiol Pharmacol 59 Suppl 6: 243-251.

5. Mackaness GB. (1964): The Immunological Basis of Acquired Cellular Resistance. J Exp Med 120: 105-120.

6. Schwartz Y, Svistelnik AV (2012): Functional phenotypes of macrophages and the M1-M2 polarization concept. Part I. Proinflammatory phenotype. Biochemistry (Mosc) 77: 246260.

7. Sica A, Mantovani A (2012): Macrophage plasticity and polarization: in vivo veritas. J Clin Invest 122: 787-795.

8. Ino Y, Yamazaki-Itoh R, Shimada K, et al. Immune cell infiltration as an indicator of the immune microenvironment of pancreatic cancer. Br J Cancer 2013; 108: 914-923.

9. Aron-Wisnewsky J, Tordjman J, Poitou C, et al. (2009): Human adipose tissue macrophages: $\mathrm{m} 1$ and $\mathrm{m} 2$ cell surface markers in subcutaneous and omental depots and after weight loss. J Clin Endocrinol Metab 94: 4619-4623.

10. Verreck FA, de Boer T, Langenberg DM, et al. (2006): Phenotypic and functional profiling of human proinflammatory type- 1 and anti-inflammatory type- 2 macrophages in response to microbial antigens and IFN-gamma- and CD40L-mediated costimulation. J Leukoc Biol 79: 285-293.

11. Van Gorp H, Delputte PL, Nauwynck HJ (2010): Scavenger receptor CD163, a Jack-of-all-trades and potential target for cell-directed therapy. Mol Immunol 47: 1650-1660.

12. Kowal K, Silver R, Slawinska E, et al. (2011): CD163 and its role in inflammation. Folia Histochem Cytobiol 49: 365-374.

13. Edin S, Wikberg ML, Dahlin AM, et al. (2012): The distribution of macrophages with a M1 or M2 phenotype in relation to prognosis and the molecular characteristics of colorectal cancer. PLoS One 7: e47045.

14. Barros MH, Hauck F, Dreyer JH, et al. (2013): Macrophage polarisation: an immunohistochemical approach for identifying M1 and M2 macrophages. PLoS One 8: e80908.

15. Vogel DY, Vereyken EJ, Glim JE, et al. (2013): Macrophages in inflammatory multiple sclerosis lesions have an intermediate activation status. J Neuroinflammation 10: 35.

16. Kunz LI, Lapperre TS, Snoeck-Stroband JB, et al. (2011): Smoking status and anti-inflammatory macrophages in bronchoalveolar lavage and induced sputum in COPD. Respir Res 12: 34 .

17. Osinska I, Wolosz D, Domagala-Kulawik J (2014): Association between M1 and M2 macrophages in bronchoalveolar lavage fluid and tobacco smoking in patients with sarcoidosis. Pol Arch Med Wewn 124: 359-364.

18. Hibbs JB Jr (2002): Infection and nitric oxide. J Infect Dis 185 Suppl 1: S9-17.
19. Cassetta L, Cassol E, Poli G (2011): Macrophage polarization in health and disease. ScientificWorldJournal 11: 2391-2402.

20. Travis WD, Costabel U, Hansell DM, et al. (2013): An official American Thoracic Society/European Respiratory Society statement: Update of the international multidisciplinary classification of the idiopathic interstitial pneumonias. Am J Respir Crit Care Med 188: 733-748.

21. Italiani P, Boraschi D. From Monocytes to M1/M2 Macrophages: Phenotypical vs. Functional Differentiation. Front Immunol 2014; 5: 514.

22. Martinez FO, Gordon S, Locati M, et al. (2006): Transcriptional profiling of the human monocyte-to-macrophage differentiation and polarization: new molecules and patterns of gene expression. J Immunol 177: 7303-7311.

23. Suttles J, Stout RD (2009): Macrophage CD40 signaling: a pivotal regulator of disease protection and pathogenesis. Semin Immunol 2009; 21: 257-264.

24. Domagala-Kulawik J, Droszcz P, Kraszewska I, et al. (2000): Expression of Fas antigen in the cells from bronchoalveolar lavage fluid (BALF). Folia Histochem Cytobiol 38: 185-188.

25. Hodge S, Hodge G, Ahern J, et al. (2007): Smoking alters alveolar macrophage recognition and phagocytic ability: implications in chronic obstructive pulmonary disease. Am J Respir Cell Mol Biol 37: 748-755.

26. Pechkovsky DV, Prasse A, Kollert F, et al. (2010): Alternatively activated alveolar macrophages in pulmonary fibrosis-mediator production and intracellular signal transduction. Clin Immunol 137: 89-101.

27. Redente EF, Higgins DM, Dwyer-Nield LD, et al. (2010): Differential polarization of alveolar macrophages and bone marrow-derived monocytes following chemically and pathogen-induced chronic lung inflammation. J Leukoc Biol 88: 159-168.

28. Wikén M, Al Hayja MA, Grunewald J, et al. (2010): No evidence of altered alveolar macrophage polarization, but reduced expression of TLR2, in bronchoalveolar lavage cells in sarcoidosis. Respir Res 11: 121.

29. Misson P, van den Brule S, Barbarin V, et al. (2004): Markers of macrophage differentiation in experimental silicosis. J Leukoc Biol 76: 926-932.

30. Renzoni E, Srihari V, Sestini P (2014): Pathogenesis of idiopathic pulmonary fibrosis: review of recent findings. F1000Prime Rep 6: 69.

31. Mills CD, Kincaid K, Alt JM, et al. (2000): M-1/M-2 macrophages and the Th1/Th2 paradigm. J Immunol 164: 61666173.

32. Mathai SK, Gulati M, Peng X, et al. (2010): Circulating monocytes from systemic sclerosis patients with interstitial lung disease show an enhanced profibrotic phenotype. Lab Invest 90: 812-823. 